\title{
CORRELATION BETWEEN HARDNESS RATIO AND DURATION OF FERMI GAMMA-RAY BURSTS
}

\author{
FU-WEN ZHANG \\ College of Science, Guilin University of Technology \\ Guilin, Guangxi 541004, China \\ fwzhang@pmo.ac.cn
}

\begin{abstract}
Using the latest gamma-ray bursts (GRB) observed by Fermi-GBM, we perform a statistical analysis of the relation between hardness ratio $(H R)$ and duration $(\Delta T)$ of GRBs, and find that $H R$ and $\Delta T$ are still anti-correlated. we also find that this correlation strongly depends upon the spectral shape of GRBs and energy bands, and the bursts with the curved spectra in the typical BATSE energy bands show a tighter anti-correlation than those with the power-law spectra in the typical BAT energy bands. This might explain why the $H R-\Delta T$ correlation is not evident for Swift-BAT GRB sample as well as other GRB sample detected by instruments with a narrower/softer energy bandpass such as HETE-2.
\end{abstract}

Keywords: Gamma-ray bursts; statistical.

PACS numbers: 95.85.Pw

\section{Introduction}

The classification of gamma-ray bursts (GRB) is still a basic open question. ${ }^{1}$ According to the traditional schemes, GRBs can be divided into long and short ones, based on the well-known bimodal distribution of their durations, which also show different spectral hardness ratios ${ }^{2}(H R)$. The $H R$ in conjunction with the duration provides a means for classification (i.e., short/hard and long/soft). However, the hardnessduration anti-correlation is not very clear in observation for some detectors with a narrow/softer energy bandpass such as HETE-2 and Swift ${ }^{3}$. The spectral hardness ratio is an important discriminator for the short/hard - long/soft dichotomy classification scheme. It is urgent to study the correlation between hardness ratio and duration further in the Fermi era. In this work, we focus attention on whether the hardness-duration anti-correlation is robust, as well as the dependence of this correlation on spectral shapes of GRBs and energy bands.

\section{Hardness Ratio and Duration}

The Fermi-GBM ${ }^{4}$ had detected 438 GRBs by the end of March 2010. The spectral properties of these GRBs have been analyzed and published by Nava et al. (2011, 
hereafter N11). ${ }^{5}$ The detailed data extraction and analysis can be found in N11. Such a catalogue is used in this work, where 316 GRBs with the curved spectra (Band or cutoff power-law model) represent sample 1, and 108 GRBs with the single power-law spectra represent sample 2. Following N11 we take the integrated time interval $(\Delta T)$, during which the signal of the GRB is above the average background, as the duration of the GRB. We note that this is not the $T_{90}$ parameter typically used to characterize the GRB duration,but the difference does not affect the statistical result.

Using the spectral data in N11, we are able to calculate HR in arbitrary two energy bands. HR in the several typical energy bands, including G1 (between the 50-100 keV and the 25-50 keV bands, typical BAT energy bands), G2 (100-300 $\mathrm{keV}$ and 50-100 keV, typical BATSE energy bands), G3 (300-1000 keV and 10-300 $\mathrm{keV})$ and $\mathrm{G} 4(100-1000 \mathrm{keV}$ and $25-100 \mathrm{keV})$, are calculated. We find that $\mathrm{HR}$ are significantly different in these energy bands for a GRB, which are expected since that the HR strongly depend on the energy bands. The left panel of Figure 1 shows $\mathrm{HR}$ versus $\Delta T$ for $424 \mathrm{GRBs}$ from N11. From this figure, we find that there is an obvious tendency that short GRBs have harder spectra than long GRBs. The correlations between $H R$ and $\Delta T$ are also analyzed in detail. We find (1) for all 424 GRBs, $H R$ and $\Delta T$ are all anti-correlated in the G1-G4 energy bands, but the correlation coefficients as well as the slopes are different; (2) the values of $H R$ are larger and the $H R-\Delta T$ anti-correlation is stronger in the typical BATSE energy bands (the median value of $\mathrm{HR}$ is 2.21 , the correlation coefficient is $r=-0.42$, the chance probability is $p=1.3 \times 10^{-19}$, and the slope is $\left.b=-0.13\right)$ than those in the
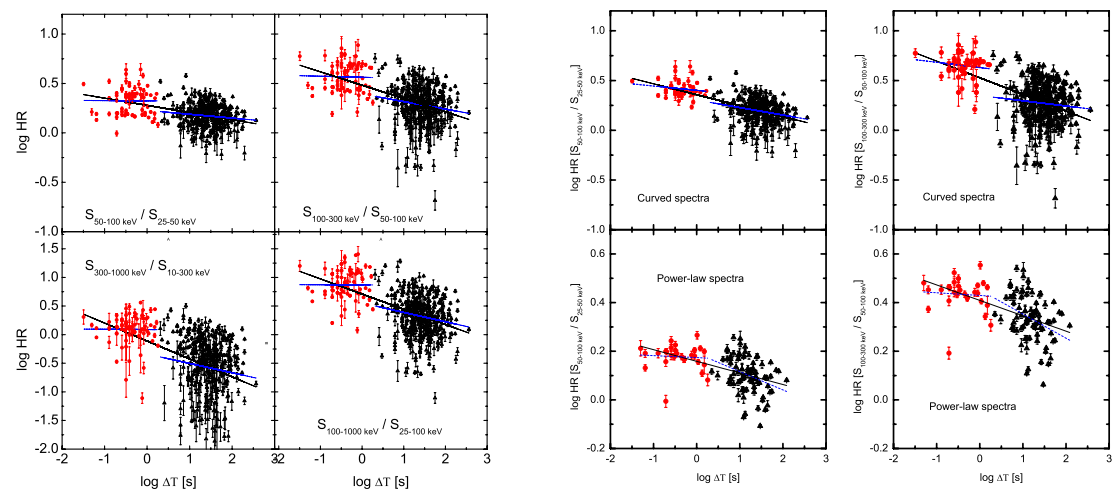

Fig. 1. Hardness ratio versus duration for all 424 Fermi GRBs (left panel) and for GRBs with the curved spectra (sample 1) or power-law spectra (sample 2) (right panel). The solid lines are the best fits for both long and short bursts, the dashed lines are the best fits to short or long events individually. 
BAT energy bands (the median value of $\mathrm{HR}$ is $1.55, r=-0.29, p=5.1 \times 10^{-10}$, and $b=-0.13$ ); (3) for short and long GRBs separately, the correlations are very weak or even negligible.

In addition, there is another question whether the $H R-\Delta T$ anti-correlation of GRBs depends on spectral shapes. For a comparison with the previous results from the BASTE and BAT observations, the $H R-\Delta T$ correlations of GRBs with the curved and power-law spectral shapes in the G1 and G2 energy bands are investigated. The results are shown in the right panel of Figure 1. We find that the anti-correlation between $H R$ and $\Delta T$ indeed depends on the spectral shape of GRBs, and the GRBs with the curved spectra have a more clear correlation $\left(r=-0.47, p=1.8 \times 10^{-19}\right.$, and $b=-0.11$ for the G1 bands, $r=-0.38, p=$ $2.3 \times 10^{-12}$, and $b=-0.17$ for the G2 bands, $\left.N=316\right)$ than those with the power-law spectra $\left(r=-0.57, p=2.2 \times 10^{-10}\right.$, and $b=-0.05$ for the G1 bands, $r=-0.57, p=2.2 \times 10^{-10}$, and $b=-0.06$ for the G2 bands, $\left.N=108\right)$ in the same energy bands. Now, the fact that there is no obvious correlation between HR and $\Delta T$ in the Swift-BAT sample as well as other samples detected by instruments with a narrow/softer energy bandpass such as HETE- $2^{2}$ can be straightforward understood. This is because (1) the $H R-\Delta T$ anti-correlation depends upon the energy bands and the correlation in the typical BAT energy bands is weak; (2) the majority of spectra of BAT GRBs can be well fitted by a single power-law model due to BAT only covers a narrow energy band, and the $H R-\Delta T$ anti-correlation for GRBs with the power-law spectra is unobvious; (3) for a given GRB sample, the values of $H R$ are small and $\Delta T$ is large for BAT-like softer detector, this makes the $H R-\Delta T$ anti-correlation unclear.

In conclusion, the anti-correlation between spectral hardness ratio and duration is confirmed by Fermi-GBM GRBs, but the correlation depends upon the spectral shapes of GRBs and energy bands. We also find out that this correlation is only a general trend between two clusters of GRBs or two types of GRBs and does not apply to either type.

\section{Acknowledgments}

We are grateful to L. Nava for providing the published Fermi-GBM data in their work. This work was supported by the National Natural Science Foundation of China (11163003) and the Guangxi Natural Science Foundation (No. 2010GXNSFB013050).

\section{References}

1. B. Zhang, Comptes Rendus Physique, 12, 206 (2011).

2. C. Kouveliotou et al., Astrophys. J. 413, L101 (1993).

3. T. Sakamoto, et al., Astrophys. J. Suppl. Ser. 195, 2 (2011).

4. W. B. Atwood, et al., Astrophys. J. 697, 1071 (2009).

5. L. Nava, G. Ghirlanda, G. Ghisellini and A. Celotti, Astron. Astrophys. 530, 21 (2011). 